\title{
Rosensweig instability in ferrofluids
}

\author{
E.I. Kats \\ Landau Institute for Theoretical Physics, RAS \\ Kosygina, 2, Moscow 119334 ,Russia \\ E-mail: kats@ill.fr; kats@landau.ac.ru \\ Laue-Langevin Institute, Grenoble F-38042, France
}

Received December 1, 2010

\begin{abstract}
We propose a simple model to analyze stability of the free surface of horizontally unbound ferrofluid in a vertical magnetic field. With respect to the well known Rosensweig instability (see e.g., R.E.Rosensweig, Ferro hydrodynamics, Cambridge University Press, Cambridge (1993) and references therein) we go one step further to include into consideration coupling of surface displacements to non-magnetic degree of freedoms. We show that the coupling can lead to a considerable reduction of the critical magnetic field and as well yields to nontrivial depletion layering near the surface.
\end{abstract}

PACS: 47.20.Ma Interfacial instabilities; 75.50.Mm Magnetic liquids.

Keywords: ferrofluids, Rosensweig instability.

Fluids with ferromagnetic properties (termed traditionally as ferrofluids) are formed by a colloidal suspension of solid magnetic particles in a parent fluid. When a layer of such a liquid is subjected to a vertically oriented and uniform magnetic field, above a critical value of the field strength a pattern of periodic peaks appears on the surface of the liquid. This is the classical Rosensweig instability observed long ago by Cowley and Rosensweig [1]. Physics behind the Rosensweig instability is related to a feed back from the ferrofluid to the applied magnetic field. This feed back modifies the magnetization drastically and establishes a new equilibrium state of the fluid. This is a typical symmetry breaking phenomenon omnipresent in the realm of phase transitions. Therefore the theoretical tools developed for thermodynamic phase transitions can be utilized to describe the Rosensweig instability. Note in passing that it is not the case for some other instabilities also known in liquids. For instance Rayleigh-Taylor or Kelvin-Helmholtz instabilities occurring as results of acceleration or shearing of liquid interfaces, are basically dynamic in their nature.

The arrangement of peaks resulting from the Rosensweig instability is a particular example of pattern formation in physical systems [2]. For these phenomena, at least as the first step, Landau phenomenological theory is an appropriate theoretical tool. Because we are dealing with a sort of instability occurring at a finite wave vector, we utilize so-called weak crystallization Landau theory $[3,4]$. The instability threshold itself can be obtained easily from a harmonic part of the surface energy. It includes two contri- butions stabilizing the flat surface, namely the surface tension term $\propto \sigma(\nabla h)^{2}$ and gravitation energy $g \rho h^{2}$ ( $\sigma$ is surface tension, $\rho$ is ferrofluid mass density, $g$ is the Earth gravitation acceleration, and $h$ is the vertical displacement with respect to the planar on average surface). On the contrary the vertical magnetic field $B$ destabilizes the flat surface contributing to the energy density as $\propto-\chi B^{2}|\nabla h|$, where $\chi$ is ferrofluid magnetic susceptibility. Combining all terms together and transforming to Fourier space ( $\mathbf{q}$ is the wave vector within the surface which we assume on average stretched along $X-Y$ axis) we arrive at the following harmonic energy density (i.e., the surface energy per unit area)

$$
a(q)=\left(\sigma q^{2}+\rho g-\chi B^{2} q\right)|h(q)|^{2} .
$$

From the (1) we find the instability threshold $B_{c}$

$$
B_{c}=\left(\frac{4 \sigma g \rho}{\chi^{2}}\right)^{1 / 4},
$$

and the optimal wave vector $q_{0}$

$$
q_{0}=\frac{\chi B_{c}^{2}}{2 \sigma}=\sqrt{\frac{g \rho}{\sigma}},
$$

surprisingly independent of ferrofluid magnetic characteristics.

The Fourier component $h\left(q_{0}\right)$ plays the role of the Landau theory order parameter $\psi$ for the Rosensweig 
instability. If one is interested not only in the threshold $B_{C}$ but also in various pattern selection, the non-linear terms have to be added to the Eq. (1). Then the standard Landau functional for the order parameter $\psi$, borrowed from the weak crystallization theory, can be written as

$$
E=\int d x d y\left\{\frac{1}{2} \alpha \psi^{2}+\frac{\beta}{8 q_{0}^{2}}\left[\left(\nabla^{2}+q_{0}^{2}\right) \psi\right]^{2}+\frac{\mu}{6} \psi^{3}+\frac{\lambda}{24} \psi^{4}\right\}
$$

The functional gives the energy of the surface bending fluctuations with wave vectors near $q_{0}$ (it determines the pattern period $2 \pi / q_{0}$ ). The first term in the Eq. (4), as usually within Landau theory, reads as $a \propto B-B_{\star}$, where $B_{\star}$ is close to the critical magnetic field $B_{C}$ (but does not coincide with it, since in a general case we have to deal with the first order phase transition). The second term has its minimum at $q=q_{0}$ and guarantees that only fluctuations with wave vectors close to $q_{0}$ are relevant. The last terms in the Eq. (4) are usual cubic and fourth-order terms in the Landau expansion relevant near the critical field. This energy (4) treats the Rosensweig "condensed ripple" formation as a pure static (thermodynamic) phase transition.

Since the Rosensweig instability we are investigating, is an athermal one, and characteristics scales (in real space) can be very large (up to $1 \mathrm{~cm}$ ), fluctuations of the order parameter are practically irrelevant. Therefore we can restrict ourselves to the mean field treatment of the energy (4). In this approximation the phase diagram can be found easily by the straightforward minimization of the (4) and the results are well known, see e.g., [4], and the Fig. 1

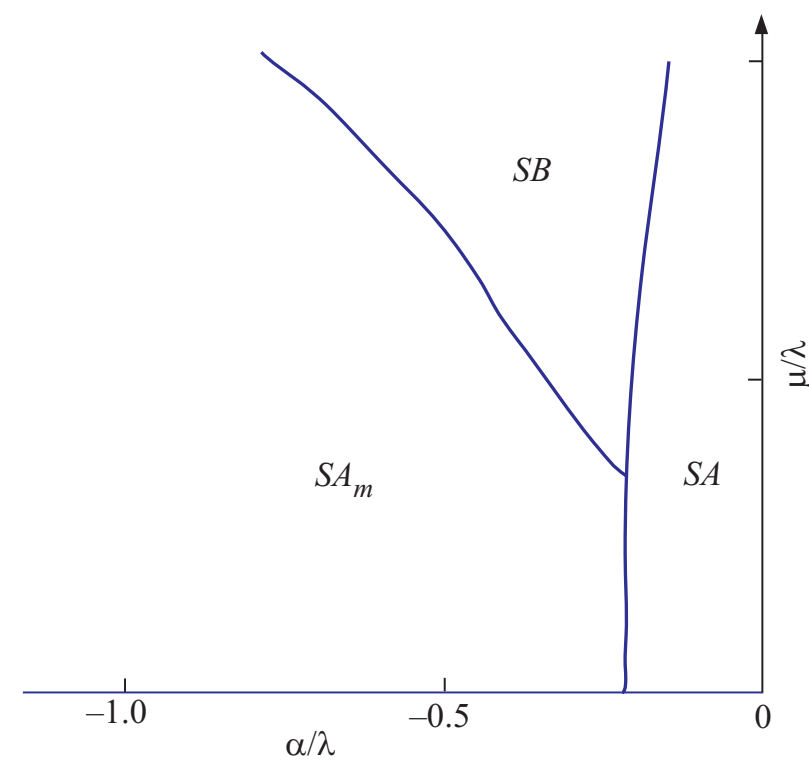

Fig. 1. The mean field phase diagram on the plane $\mu / \lambda$ and $\alpha / \lambda$. SA stands for the flat surface, $S B$ for the hexagonal structure, and in the $S A_{m}$ phase one dimensional modulation takes place. where for the case $\lambda=$ const and $\mu=$ const the phase diagram is plotted on the plane $\mu / \lambda$ and $\alpha / \lambda$. This is the phase diagram when the both non-linear (interaction) terms coefficients $\mu$ and $\lambda$ in the Landau energy (4) are constant, independent of wave vectors. This describes the first instability with hexagonal pattern formation. However, experimentally upon further increase of the magnetic field above the threshold $B_{C}$ gives rise to the transition from the hexagonal to square lattice of peaks [5]. Weak crystallization Landau theory is equally suitable to describe this second instability. Indeed, as we already mentioned above, neglecting fluctuations is equivalent to fixing $|\mathbf{q}|=q_{0}$. In this case (i.e., in the mean field approximation) $\mu$ indeed has to be considered as a constant. However $\lambda$ is allowed to be a function of a single angle $\theta$ between the four wave vectors entering into the fourth order term. These wave vectors have to satisfy the conditions

$$
\left|\mathbf{q}_{i}\right|=q_{0} ; \quad \sum_{i=1}^{4} \mathbf{q}_{i}=0,
$$

and in the most general form it means that

$$
\lambda=\lambda_{0}\left(1+\sum_{k} \lambda_{k} \cos (2 k \theta)\right),
$$

where $\lambda_{k}$ describe anisotropy of the fourth order interaction. Keeping only $\lambda_{1} \neq 0$ we can obtain the phase diagram (see Fig. 2) which includes also a tetragonal phase.

However recent experimental observations [6] show that something is missing in this picture. This unknown something yields to a considerable reduction of the critical field, and as well produces non-trivial depletion layering near the surface. Reduction of the critical field means that there is another destabilizing factor promoting surface undulations. Pure phenomenologically depletion and nonuniform colloidal particle distribution can be bluntly lumped into an effective field $\phi$ coupled to the surface curvature $\nabla^{2} h \equiv \nabla^{2} \psi$. In a Fourier space

$$
E_{\text {int }}=\gamma \phi(q)\left(q^{2} \psi(-q)\right) .
$$

In the spirit of the Landau theory the energy per unit area responsible for colloid composition variations, reads as

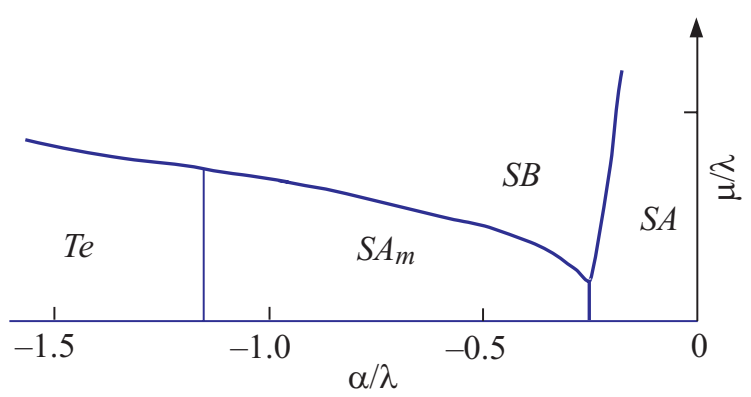

Fig. 2. The phase diagram with anisotropic $\lambda$ with a tetragonal structure $T e$. 


$$
E_{\mathrm{comp}}=\int d x d y\left\{\frac{1}{2} t \phi^{2}+\frac{c}{2}(\nabla \phi)^{2}+\frac{d}{4} \phi^{4}\right\}
$$

Within the mean field approximation minimizing $E_{\text {comp }}+E_{\text {int }}+a(q)$ over $\psi(q)$ we get

$$
\psi(q)=\frac{\gamma \phi_{q} q^{2}}{\sigma q^{2}+g \rho-\chi B^{2} q} .
$$

Then we find the effective free energy in terms of $\phi$ with the quadratic over $\phi(q)$ term $\Gamma_{q}|\phi(q)|^{2}$ with

$$
\Gamma_{q}=t+c q^{2}-\frac{\gamma^{2} q^{4}}{\sigma q^{2}+g \rho-\chi B^{2} q},
$$

with its minimum at $q_{0}^{2} \neq 0$ if $\gamma^{2} \geq \gamma_{0}^{2} \neq 0$. Note that this instability might occur even at $B=0$. In this simple model in a single mode approximation there are three equilibrium phases:

- flat and symmetric phase $(\langle\phi\rangle=0$ and $\langle\psi\rangle=0)$;

- flat and asymmetric phase $(\langle\phi\rangle \neq 0$ and $\langle\psi\rangle=0)$;

- asymmetric and modulated phase $(\langle\phi\rangle \neq 0$, and $\langle\psi\rangle \neq 0)$.

Our assumptions of the "weak crystallization» nature of the phase transition should be treated as a working hypotheses. Comparison of the predictions resulting from this hypothesis with experimental observations will show whether and when this hypothesis is justified. While the picture is still not completely clear we do believe that further detailed studies of this transition, both from the experimental and theoretical sides, will increase our understanding of the mechanisms enabling ferrofluids to accommodate different structures and physical properties.

I am very pleased to dedicate this article to $\mathrm{V}$. Peschanskii on the occasion of his 80-th birthday. He certainly loves "Electron phenomena in conducting systems", the topic of this special issue, but as well condensed matter physics in general, and discussions with him have been an inspiration for this paper.

The author acknowledge support from Russian Federal grant "FTP Kadry".

1. M.D. Cowley and R.E. Rosensweig, J. Fluid Mech. 30, 671 (1967).

2. M.C. Cross and P.H. Hohenberg, Rev. Mod. Phys. 65, 851 (1993).

3. S. Brazovskii, Sov. Phys. JETP 41, 85 (1975).

4. E.I. Kats, V.V. Lebedev, and A.R. Muratov, Phys. Rep. 228, 1 (1993).

5. R. Friedrichs and A. Engel, Phys. Rev. E64, 021406 (2001).

6. A. Vorobiev, G. Gordeev, O. Konovalov, and D. Orlova, Phys. Rev. E79, 031403 (2009). 